\title{
MOBILE UNDERWATER DEBRIS SURVEY SYSTEM (MUDSS)
}

\author{
P.J. Carroll , J. D. Lathrop, J.F. McCormick, and D.C. Summey \\ Coastal Systems Station, Dahlgren Division, Naval Surface Warfare Center, Code R23 \\ Panama City, FL 32407-5000 \\ (850) 234-4795, Carroll_Paul@ccmail.ncsc.navy.mil \\ T.-H. Chao, A. Chutjian, and J. Wright \\ Jet Propulsion Laboratory \\ Pasadena,CA $91109-8099$
}

\begin{abstract}
This paper reports on the development of MUDSS, a multi-sensor system for the surveying of underwater ordinance and explosive waste (OEW) in shallow water $\left(10^{\prime}\right.$ to $\left.200^{\prime}\right)$. The MUDSS concept and mode of operation are described and operational performance of the MUDSS state-of-the-art (SOA) sensors is presented from preliminary tests against a range of OEW targets. MUDSS employs advanced real-time processing and data fusion techniques to improve target detection in the presence of clutter, a significant problem in many shallow water sites of interest. MUDSS is expected to provide a substantial and unmatched capability to accurately survey uriderwater sites as the first step to remediation
\end{abstract}

\section{INTRODUCTION}

MUDSS is a four-year Strategic Environmental Research and Development Program (SERDP) project to develop and demonstrate a system which can successfully survey underwater formerly used defense sites for ordinance and explosive waste (OEW). MUDSS is being executed jointly by the Navy's Coastal Systems Station (CSS) and by the Jet Propulsion Laboratory (JPL). MUDSS heavily leverages (1) SOA acoustic, magnetic and electro-optic (EO) minehunting sensors and signal and image processing technologies developed by CSS for the Office of Naval Research, (2) signal and image processing techniques and visualization technologies under development at JPL for the National Aeronautics and Space Administration and (3) trace chemical detection technologies under development at JPL for the Federal Aviation Administration.

Selected sensor and processing elements of MUDSS have been successfully tested in a MUDSS deployment configuration against $\mathrm{OEW}$ targets using on-hand acoustic, magnetic and EO sensors similar or identical to the final MUDSS sensor suite. Test results showed that the proposed MUDSS sensors show good promise against OEW targets and are well characterized by sensor performance models already in place at CSS. Reference 1 provides details on MUDSS development and testing beyond that reported here.

\section{DESCRIPTION OF MUDSS}

A conceptual view of MUDSS is shown in Fig. 1. The surface craft is a custom designed, magnetically and acoustically quiet, shallow draft, trailerable catamaran. An active-wing depressor which is suspended off the back of the catamaran houses a RESON Seabat ahead looking sonar, a CSS/Grumman-developed dual high frequency/low frequency $(180 / 22.5 \mathrm{kHz})$ synthetic aperture sonar (HF/LFSAS), and a $532 \mathrm{~nm}$ CSS/Raytheon-developed laser linescanner (LLS). The LLS is capable of $6 \mathrm{~mm}$ resolution. Characteristics of the HF/LFSAS and Seabat sonars are in Table 1. The depressor also houses processing electronics and a motion measurement package to optimize sensor operation. A neutrally bouyant body housing a superconducting magnetic gradiometer is towed 50' behind the depressor.

The depressor's active-wing controls the depth of the

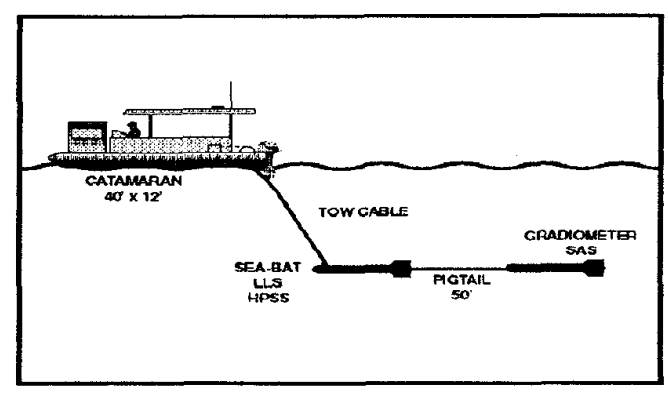

Fig. 1. Sketch of the MUDSS 
towed system. A cabin onboard the catamaran provides space for sensor operators and the control and data acquisition electronics. The cabin and the catamaran's 10 $\mathrm{kW}$ generator are placed near the front of the catamaran to maximize the distance from these two strong magnetic objects to the magnetic gradiometer. MUDSS also includes a chemical sensor, not shown in Fig. 1, housed in a separate vehicle which can be deployed to collect sea bottom samples near suspected targets which are analyzed to detect trace explosive elements.

\section{MUDSS MODES OF OPERATION}

MUDSS is intended to provide detection of a range of OEW objects from $2000 \mathrm{lb}$ bombs to $60 \mathrm{~mm}$ munitions in a variety of shallow water environments. The MUDSS concept and mode of operation draw heavily from sensor and underwater target detection experience of Navy developers familiar with a closely related problem, minehunting in shallow water. Underwater target detection and identification is fundamentally difficult. Targets of interest vary in size with dimensions of a few feet to a few inches. Targets can be proud or may be buried in the bottom. There are potentially numerous extraneous underwater objects which appear acoustically or magnetically as clutter. Multipath reverberation of acoustic signals in shallow water reduces the target signal-to-noise ratios.

The MUDSS approach is to combine the capabilities of the best SOA acoustic, magnetic and LLS sensors used in combination to detect and identify UXO and OEW targets at the highest possible survey rates.

MUDSS survey operations begin with a search phase and follow with an identification phase. In the search mode, the catamaran tows the depressor/towed vehicle above the bottom at a height which will provide the maximum detection swept path for targets of interest. The Seabat, the dual frequency SAS, and the gradiometer operate simultaneously. The acoustic images of the sea bottom are displayed to the operator on raster displays in real time. Magnetic gradiometer data is processed in real time to provide location of detected magnetic targets and

Table 1. Characteristics of the MUDSS sonars

\begin{tabular}{|l|c|c|}
\hline Sensor Type & HF/LF SAS & Seabat 6012 \\
\hline Center Frequency & $180 \mathrm{kHz} / 22.5 \mathrm{kHz}$ & $455 \mathrm{kHz}$ \\
\hline Azimuth Resolution & $2.5 \mathrm{~cm} / 7.5 \mathrm{~cm}$ & $1.5^{\circ}$ \\
\hline Range Resolution & $2.5 \mathrm{~cm} / 7.5 \mathrm{~cm}$ & $5 \mathrm{~cm}$ \\
\hline Range @ 8 Knots & $2 \mathrm{~m}-45 \mathrm{~m}$ & $5-200 \mathrm{~m}$ \\
\hline Number of Beams & $9 / 3$ & $\begin{array}{c}60 \\
\left(90^{\circ} \text { sector }\right)\end{array}$ \\
\hline
\end{tabular}

magnetic moment information. Data fusion techniques are applied to the acoustic and magnetic data to determine if observed targets are both acoustically and magnetically detected at the same location (after acoustic and magnetic sensor separation distances are compensated). Detected target positions are catalogued by GPS position on board the catamaran. Most OEW targets of interest are expected to have both an acoustic and magnetic detectable signal. The catamaran travels at 8 knots. A swept path of 100 meters would be expected for large magnetic target (2000 $\mathrm{lb}$ bomb) with magnetic sensor operating at a sensitivity of $3 \mathrm{pT} / \mathrm{m}$. This would permit surveying approximately 0.4 square nautical miles per hour.

The LLS sensor is the primary sensor in the target identification mode. Targets are reacquired using GPS coordinates from the detection phase and using the wide angle Seabat to assist in reacquisition. The objective is to fly over the target at close ranges to obtain a visual image confirming target identification. If target visual identification is not possible the system includes a separate AUV which will sample soil in the vicinity of the target to attempt to detect chemical traces of explosives. Chemical analysis of these samples is performed back in the laboratory.

The final product of a MUDSS survey is a map providing precise location of the detected OEW. The survey is expected to form the basis of remediation action or to be used to monitor changes in OEW location with time.

\section{MUDSS SENSOR PERFORMANCE TESTING}

The MUDSS program objective is to complete development of the MUDSS sensor suite and perform OEW survey demonstration operating with real-time sensor and data fusion processing. The survey demonstration will be in late FY98 in Choctawhatchee Bay near Eglin AFB, Florida where practice bombs were dropped during training missions.

The performance of several MUDSS component sensors was demonstrated during a test in St. Andrews Bay in 1995. The sensor suite included a Seabat, an SAS sensor, a gradiometer, and Raytheon's line scanner LS 4096. Selected sensor test results are presented here to support estimates of MUDSS operation presented above. Test results also presented an opportunity to confirm that CSS acoustic, magnetic and EO computer models accurately predict observed results.

MUDSS tests were performed using target fields deployed in 30 feet of water in a sand bottom. The targets ranged in size from $60 \mathrm{~mm}$ motar shells to $2000 \mathrm{lb}$ bombs. 


\section{ACOUSTIC RESULTS}

The acoustic images of the targets from the SAS were superior to what would bs: expected from a conventional side scan sonar. This was predicted by a sonar performance prediction model known as SWAT (Shallow Water Acoustics Toolset), which was developed at CSS to predict the performance of imaging sonars operating in the difficult shallow water environment. SWAT analysis was used to explain the performance of the test sensors against the target fields. The SWAT validation enables MUDSS to confidently predict the performance of updated SAS to be used in the final MUDSS configuration and compare expected results versus other commercial sonars.

Fig. 2 shows images of a $1000 \mathrm{lb}$ bomb target and a 203 $\mathrm{mm}$ howitzer from a selected test. The top images from the SAS show sharp and detailed highlights from both targets. The middle images simulated by SWAT show high fidelity agreement between the predicted and actual images. The lower images are corresponding images simulated by SWAT for a high- quality commercial 100 $\mathrm{kHz}$ commercial side scan sonar. The SAS target images are clearly superior to the predicted side scan images.

\section{MAGNE]:ICS RESULTS}

The gradiometer tested for MUDSS has two principal advantages over simpler, more common magnetic field sensors such as total field magnetometers. First, it has the highest known sensitivity of any field-deployable magnetic sensor, providing it with excellent range (over $50 \mathrm{~m}$ against a $2000 \mathrm{lb}$ bo:mb). Secondly, its five channels measure all of the independent components of the gradient of the magnetic field, providing sufficient information to localize simultaneously on as many as three targets. By comparison, a total field magnetometer has only one channel and cannot unambiguously localize on even a single target.

In addition to being able to find the position of multiple magnetic targets, the gradiometer also determines their magnetic moments. This magnetic classification information can then be used in conjunction with the sonar images to help correctly classify OEW targets and sort them from the surrounding debris.

A portion of the gradiometer time series from a pass near a $1000 \mathrm{lb}$ bomb is showr in Fig. 3. An automatic realtime localization algorithm operates on signals such as these to determine the locations and magnetic moments of the targets contributing to the signals. In Fig. 3, this algorithm finds a target having the magnetic moment of a $1000 \mathrm{lb}$ bomb located $15 \mathrm{~m}$ to port and $5 \mathrm{~m}$ below the gradiometer, very close to the known location of the actual bomb. Fig. 4 shows the locations of the magnetic targets found by the gradiometer in a pass over one of the target fields (the gradiometer trajectory as inferred from the GPS data can be seen in the Fig.). The size of the dot at each target location is in proportion to the size of the magnetic moment found for the target, illustrating the capability of the gradiometer to distinguish between large and small targets.

It is evident from Fig. 4 that all of the targets in the linear field, whose actual positions are marked with open circles, have been found by the gradiometer with the exception of the $1000 \mathrm{lb}$ bomb. The localization algorithm instead found a second target at the location of the $500 \mathrm{lb}$ bomb and another (false) target further on to port. Errors of this sort can arise when targets are too closely spaced.

\section{ELECTRO-OPTICS RESULTS}

The LS 4096 operating at $532 \mathrm{~nm}$, the blue/green window, uses a laser and photmultiplier receiver synchronously scanned on successive pixels to develop images at the bottom. The LS 4096 has a 70 degree field of view and provides high resolution pixels $(0.25 \mathrm{inch}$ at $4 \mathrm{kts})$. Laser line scan techniques reduce backscatter and blur/glow/ forward scatter compared to standard passive cameras.

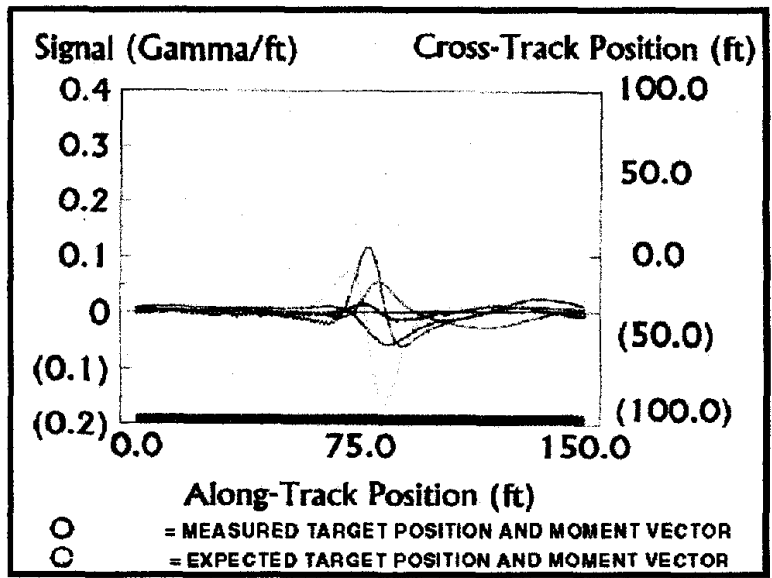

Fig. 3. The five gradiometer signals in a $150-\mathrm{ft}$ section of data on a pass near the $1000 \mathrm{lb}$ bomb in the linear target field. The signals are due principally to the bomb, although other targets in the ficld are contributing, as is evident in the tails of the signals. The measured and expected target location and moment are also displayed. The expected direction of the moment vector is determined from knowledge of the orientation and shape of the target, whose moment is mainly induced. 

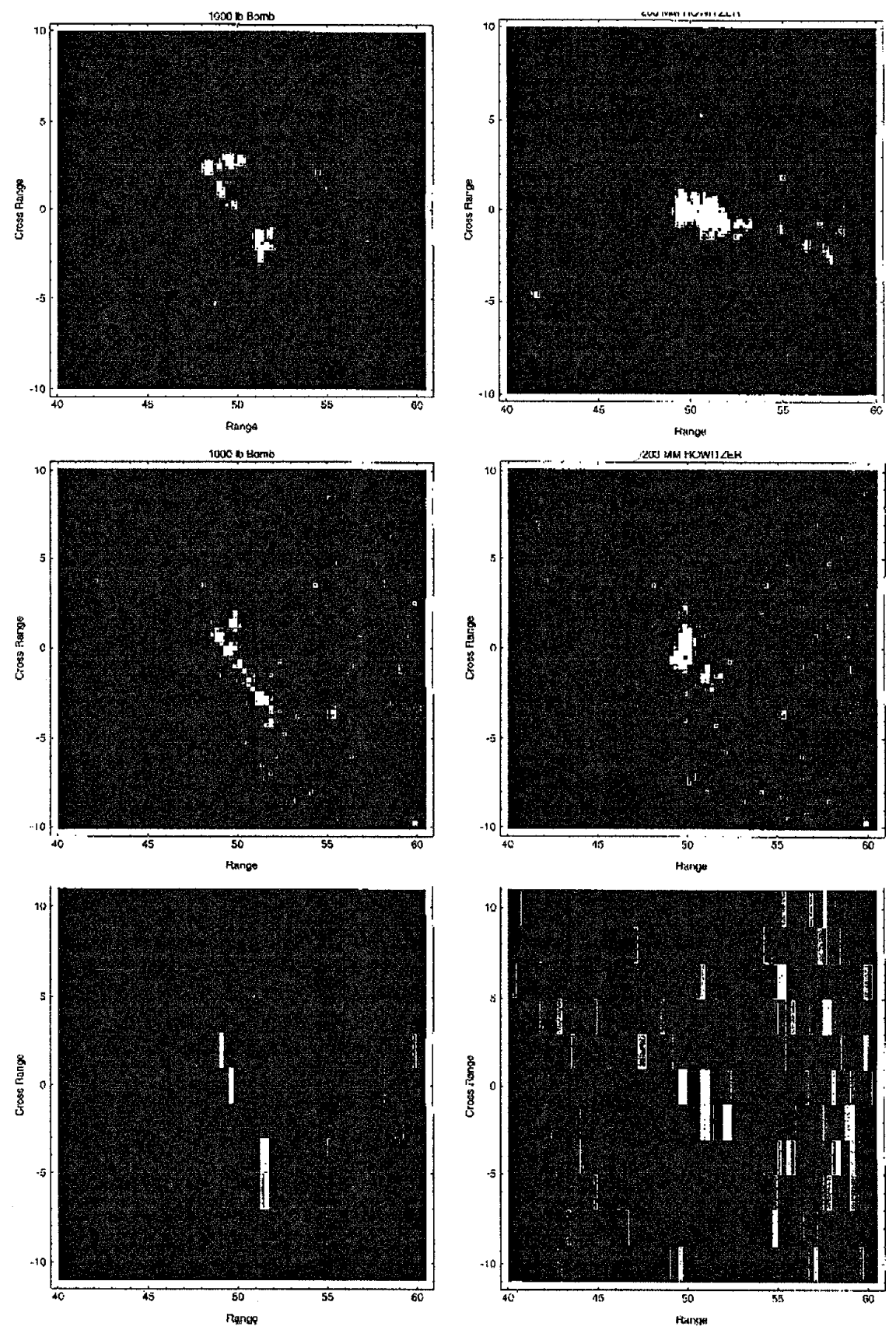

Fig.2. Images of a $1000 \mathrm{lb}$ bomb (left column) and $203 \mathrm{~mm}$ howitzer (right column) as actually seen by the SAS, as predicted by SWAT for the SAS, and as predicted by SWAT for a high-quality $100 \mathrm{kHz}$ commercial sidescan sonar under the same conditions. 


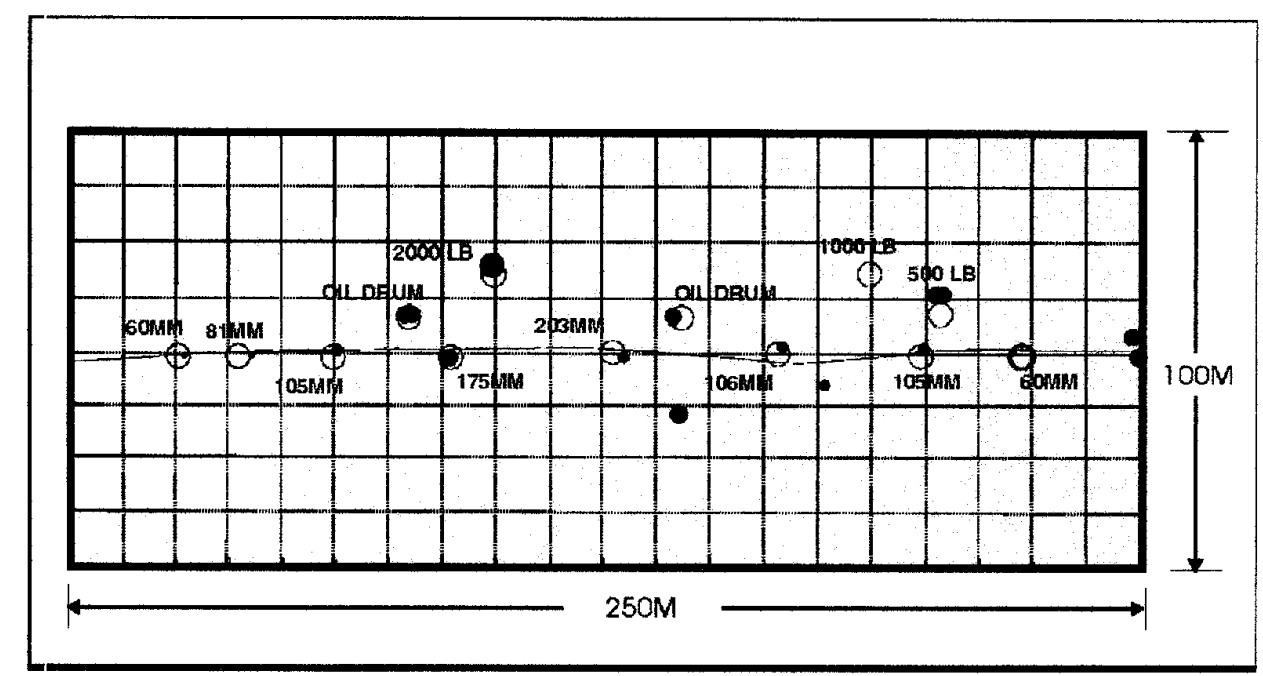

TARGET LOCATION

MAGNETIC TARGET LOCALIZATION (SIZI INDICATES MAGNETIC MOMENT)

Fig. 4. Gradiometer targets (black circles) found in Run 118 over the linear field. The size of each black circle is proportional to the size of the target moment. The open circles are the actual target locations.

Image ranges are on the order of several (3-6) attenuation lengths. Fig. 5 shows an LS 4096 image of a $60 \mathrm{~mm}$ motar shell at a range of $5 \mathrm{~m}$ ( 3.5 attenuation lengths). The image quality is excellent and sufficient for target identification.

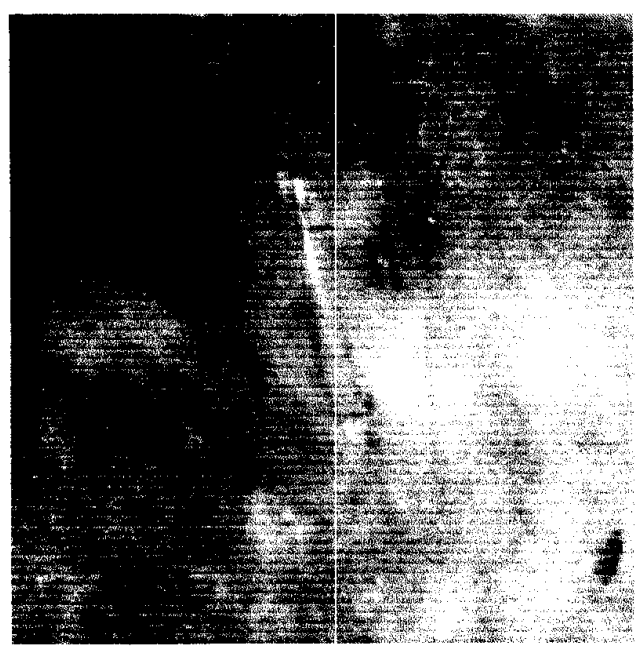

Fig. 5. LS4096 image of a $60 \mathrm{~mm}$ motar at a range of $5 \mathrm{~m}$ (3.5m attenuation lengths)

\section{CONCLUSION}

Results from MUDSS testing of on-hand Navy sensors demonstrate good detection, classification and identification capability against a wide range of OEW targets in shallow water. Real-time data fusion and signal processing capabilities are being developed in preparation for a MUDSS demonstration testing at a UXO site in late FY98. MUDSS is expected to provide a substantial new and improved capability to accurately survey underwater sites as the first step to remediation.

\section{ACKNOWLEDGMENTS}

The authors wish to thank sponsors at ONR, NASA and SERDP for long term support and the entire MUDSS development team including P.J. Bernstein, J.T. Bono, J.F. McCormick, D.J. Overway, G.S. Sammelmann at CSS and T.H. Chao and K.C. Scott at JPL. This work was performed under Project 052-NAVY of the Strategic Environmental Research and Development Program.

\section{REFERENCES}

[1] J.D. Lathrop, J.F. McCormick, P.J. Bernstein, J.T. Bono, D.J. Overway, G.S. Sammelmann, et al, "Mobile Underwater Debris Survey System (MUDSS) Feasibility Demonstration Report," UXO Forum 1996 Conference Proceedings, pp. 427-436, March 1996. 